\section{Resultados histopatológicos de segunda revisión por carcinoma urotelial vesical no invasor al músculo: ¿realmente es útil?} Chamlati-Cuello JM, Garza-Gangemi AM, Gonzaga-Carlos N, Iturriaga-Goyon
E, Castillejos-Molina RA

\section{Resumen}

ANTECEDENTES: en resecciones repetidas en cáncer de vejiga existe una alta tasa de tumor residual. En la actualidad, la verdadera utilidad y el efecto de este tratamiento son inciertos.

OBJETIVO: describir los resultados de la segunda revisión en pacientes con cáncer de vejiga no invasor al músculo, según la tasa de re-estadificación y coexistencia de tumor residual.

MATERIALES Y METODOS: estudio retrospectivo y observacional, efectuado en pacientes con resección transuretral de vejiga por carcinoma urotelial vesical no invasor al músculo, de 1984 a 2017, en el Instituto Nacional de Ciencias Médicas y Nutrición Salvador Zubirán. Se analizó la tasa de tumor residual y re-estadificación en la segunda revisión.

RESULTADOS: de 556 expedientes, se incluyeron para el análisis final 117 casos, de los que solo $43(37.7 \%)$ formaron parte del grupo de pacientes con segunda revisión. El diámetro del tumor primario fue mayor en el grupo de estudio ( $3 \pm 2$ vs $2.3 \pm 1.5 \mathrm{~cm}, \mathrm{p}=0.03)$, al igual que el tumor residual en 17 (39.5\%) casos y 1 (2.3\%) expresó tumor con invasión al músculo. En los pacientes con tumor residual, $2(11.7 \%)$ tuvieron un incremento en estadio y $3(17.64 \%)$ un cambio de grado bajo a grado alto.

CONCLUSIONES: existe un alto porcentaje de tumor residual en pacientes con diagnóstico de cáncer de vejiga no invasor al músculo, inicialmente tratados con resección transuretral de vejiga, además de una tasa re-estadificación considerable.

PALABRAS CLAVE: cáncer de vejiga, segunda revisión, tumor residual, re-estadificación.
Departamento de Urología, Instituto Nacional de Ciencias Médicas y Nutrición Salvador Zubirán, Ciudad de México.

Recibido: mayo 2017

Aceptado: mayo 2017

Correspondencia Jorge Mario Chamlati Cuello chamlati_md@me.com

Este artículo debe citarse como

Chamlati-Cuello JM, Garza-Gangemi AM, GonzagaCarlos N, Iturriaga-Goyon E, Castillejos-Molina RA. Resultados histopatológicos de segunda revisión por carcinoma urotelial vesical no invasor al músculo: ¿realmente es útil? Rev Mex Urol. 2017 mayo;77(3):183-190. 
Rev Mex Urol. 2017 may;77(3):183-190.

\section{Second-look histopathologic results in non-muscle-invasive urothelial carcinoma of the bladder: Is repeat resection necessary?}

Chamlati-Cuello JM, Garza-Gangemi AM, Gonzaga-Carlos N, Iturriaga-Goyon E, Castillejos-Molina RA

\section{Abstract}

BACKGROUND: There is a high rate of residual tumor reported in repeat resections performed in cases of bladder cancer. The real usefulness and effect of this practice are currently in doubt.

OBJECTIVE: To describe the results of a second-look resection in patients with non-muscle invasive bladder cancer in relation to the rates of re-staging and residual tumor.

MATERIALS AND METHODS: A retrospective, observational study was conducted on patients that underwent transurethral resection of the bladder due to non-muscle-invasive urothelial carcinoma of the bladder at the Instituto Nacional de Ciencias Médicas y Nutrición Salvador Zubirán, within the time frame of 1984 to 2017. Second-look residual tumor and re-staging rates were analyzed.

RESULTS: Out of 556 medical records, the final analysis included 117 cases, of which only 43 patients $(37.75 \%)$ underwent a second-look resection. Primary tumor diameter was larger in the study group (3 \pm 2 vs $2.3 \pm 1.5 \mathrm{~cm}, \mathrm{p}=0.03)$. Residual tumor was found in 17 cases $(39.5 \%)$, one of which $(2.3 \%)$ presented with muscle-invasive disease. In the patients with residual tumor, there was an increase in stage in 2 cases $(11.76 \%)$, and 3 cases $(17.64 \%)$ had a change from low grade to high grade tumor.

CONCLUSIONS: There is a high percentage of residual tumor in patients diagnosed with non-muscle-invasive urothelial carcinoma of the bladder that underwent initial transurethral resection of the bladder, as well as a considerable re-staging rate.

KEY WORDS: Bladder cancer; Second-look resection; Residual tumor; Re-staging
Departamento de Urología, Instituto Nacional de Ciencias Médicas y Nutrición Salvador Zubirán, Ciudad de México.

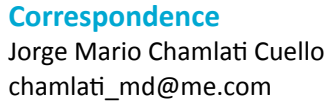

\section{ANTECEDENTES}

El cáncer de vejiga es la séptima neoplasia más frecuente diagnosticada en hombres en todo el mundo y la decimoprimera considerando ambos sexos. La incidencia reportada en hombres y mujeres es de 9 vs 2.2 por cada 100,000 personas al año, respectivamente. En América Latina la 
incidencia estimada es de 3.3 en hombres y de 1.3 en mujeres. La incidencia más alta reportada en este continente, tomando en cuenta ambos sexos, se registra en Uruguay (8.5) y la más baja en Guatemala (0.6). En México, en el año 2012 se reportó una incidencia de 3.8 en hombres y de 2 en mujeres, con una mortalidad de 1.3 y 0.3 (por cada 100,000), respectivamente. ${ }^{1}$ La incidencia del cáncer de vejiga varía según el país, factores de riesgo, prácticas de detección y disponibilidad del tratamiento. ${ }^{2}$

En 75 a $85 \%$ de los casos la enfermedad está confinada a la mucosa ( $\mathrm{Ta}$, carcinoma in situ) o submucosa (T1), lo que se denomina enfermedad no invasora al músculo. En este grupo de pacientes los tumores de vejiga no invasores al músculo de alto grado son de especial interés, pues se consideran de alto riesgo de recurrencia y, desde el punto de vista oncológico, de progresión. La resección transuretral de vejiga es el tratamiento de primera línea y de ésta depende la extirpación de un buen espécimen para efectuar el estudio histopatológico inicial y establecer la estadificación correcta de la neoplasia. En este proceso se obtiene información adicional para implementar el tratamiento inicial y sugerir el pronóstico, por ejemplo: tamaño, localización, multiplicidad, grado y coexistencia de carcinoma in situ. ${ }^{3}$ El riesgo puede estimarse de forma individual, mediante el sistema de puntajes y tablas de riesgo de las guías de la Asociación Europea de Urología. Un subgrupo de riesgo incluye pacientes con $\mathrm{T} 1$ de alto grado al momento del diagnóstico, ya que son difíciles de tratar y tienen alto potencial de malignidad asociada con pobre pronóstico, comparados con otros tumores no invasores de músculo. De hecho, el límite de progresión varía de 22 a 33.3\% y la mortalidad por cáncer específica es de 12 a 15\%.

En $50 \%$ de los pacientes con $\mathrm{T} 1$ es posible la preservación vesical mediante resección transuretral e inmunoterapia vesicales. Sin embargo, se ha demostrado que algunos pacientes tratados de esta manera permanecen enfermos debido a: errores de estadificación, evaluación histopatológica incorrecta del tumor, biopsias obtenidas al azar del urotelio macroscópicamente sano, calidad de la resección, diferente historia natural de los tumores, tiempo ideal no estandarizado para considerar la cistectomía o radioterapia, y asumimos que los tumores T1, per se, tienen un comportamiento heterogéneo. ${ }^{4}$ Entre las ventajas de la cistectomía radical se encuentran su bajo riesgo de recurrencia (siempre y cuando la realice un médico con experiencia en este tipo de intervención), que puede efectuarse en pacientes de edad avanzada; el riesgo en centros hospitalarios donde se lleva a cabo este procedimiento poco puede variar. En este punto, la aplicación de BCG (Bacilo de Calmette y Guérin) tiene función importante en la preservación vesical. ${ }^{5}$

La estrategia de "segunda revisión" se introdujo en 1990 debido a los hallazgos reportados en resecciones repetidas de tumor residual en $33.8-60 \%$ de los casos y enfermedad con invasión al músculo en 5 a $20 \%$ de los pacientes con enfermedad T1 inicial. La probabilidad de invasión al músculo puede elevarse a $45 \%$ en caso de no detectarla en la primera muestra. Esto sugiere que la mayor parte de los tumores de alto grado se resecan de manera incompleta y requieren una nueva cirugía para establecer la estadificación y tratamiento adecuados. En la actualidad, múltiples guías (EAU, AUA) recomiendan efectuar una segunda revisión en pacientes que inicialmente tuvieron resección incompleta, ausencia de músculo en la pieza quirúrgica (excepto los casos de TaG1 y carcinoma in situ primario), se estadificaron con T1 y en quienes se diagnosticaron tumores de alto grado. El tiempo recomendado para realizar la segunda revisión varía de 2 a 6 semanas después de efectuar la primera, intervalo que surgió de manera inicial como recomendación del Consenso y, posteriormente, fue surgiendo evidencia 
de la repercusión oncológica de este periodo, en términos de recurrencia y progresión de la enfermedad. ${ }^{6-7}$ Algunos estudios demuestran un efecto positivo en los resultados oncológicos de la segunda revisión. En pacientes con seguimiento a cinco años se han observado efectos satisfactorios de la segunda revisión en cuanto a recurrencia (39.8 vs $71.4 \% ; \mathrm{p}=0.0001$ ) y progresión de la enfermedad (6.5 vs 23.5\%; $\mathrm{p}=0.001) .{ }^{8}$ En contraparte, existen grupos que discuten su utilidad, pues sugieren que no existe diferencia en la tasa de recurrencia, progresión y mortalidad por cáncer. ${ }^{9}$ Actualmente no existe información contundente para sistematizar esta práctica; por lo tanto, la verdadera utilidad y el efecto terapéutico de la segunda revisión puede considerarse incierto.

El objetivo de este estudio es describir los resultados de la segunda revisión en pacientes con cáncer de vejiga no invasor al músculo, de acuerdo con la tasa de re-estadificación y coexistencia de tumor residual.

\section{MATERIALES Y MÉTODOS}

Estudio de cohorte, prospectivo, al que se incluyeron pacientes intervenidos de resección transuretral de vejiga, entre julio de 1984 y febrero de 2017, en el Instituto Nacional de Ciencias Médicas y Nutrición Salvador Zubirán. Criterios de inclusión: pacientes con diagnóstico inicial de cáncer de vejiga no invasor al músculo, de 18 años de edad o mayores. Criterios de exclusión: pacientes con diagnóstico de cáncer invasor al músculo, resección transuretral incompleta de vejiga e información inconclusa en el expediente durante su seguimiento.

Para el análisis de los datos los pacientes se dividieron en dos grupos: quienes fueron objeto de segunda revisión (Grupo 1) y a quienes no se les realizó (Grupo 2). La segunda revisión se define como la resección transuretral efectuada después de establecer el diagnóstico inicial, según los criterios de las guías internacionales del tratamiento de cáncer de vejiga no invasor al músculo: tumores en estadío T1 o de alto grado, y ausencia de músculo en el resultado histopatológico inicial (excepto en TaG1 y coexistencia de carcinoma in situ) o resecciones incompletas. En ambos grupos se analizaron las características basales (edad, sexo, tabaquismo e IMC), comorbilidades (diabetes tipo 2, hipertensión arterial sistémica, dislipidemia y enfermedad renal crónica) y tipo de hematuria (macro y microhematuria), además de las características patológicas como: diámetro tumoral $(\mathrm{cm})$, estadificación T y grado de diferenciación. En los pacientes con segunda revisión se analizaron: la coexistencia de tumor residual, infraestadificación, cambio del grado tumoral y enfermedad músculo-invasora. Por último, se registró la indicación de tratamiento coadyuvante (BCG o mitomicina $\mathrm{C}$ ) y los desenlaces oncológicos (tasa de recurrencia y progresión). Se definió recurrencia tumoral a la demostración histopatológica de tumor viable en la resección transuretral de vejiga o la biopsia durante el seguimiento del paciente y como progresión al aumento en el grado tumoral o estadificación respecto del diagnóstico inicial.

Para el análisis estadístico se utilizó el programa SPSS 21 para IBM. El análisis descriptivo incluyó medidas de tendencia central (media o mediana) y de dispersión (desviación estándar o intervalo intercuartil, según la distribución de la variable estudiada). La distribución de variables se evaluó con la prueba de Shapiro-Wilk y el análisis bivariado con la t de Student para muestras independientes en variables paramétricas y $U$ de Mann-Whitney para variables no paramétricas. Las variables cualitativas se analizaron con $\chi^{2} y$ prueba exacta de Fisher. Se consideró estadísticamente significativo el valor de $p \leq 0.05$ o $5 \%$ para una distribución de dos colas. 


\section{RESULTADOS}

Se revisaron 556 expedientes de pacientes con resección transuretral de vejiga inicial, de los que solo 117 cumplieron con los criterios de inclusión: $43(37.7 \%)$ asignados al grupo de segunda revisión (Grupo 1). La edad media al diagnóstico fue de 68 años. Se registraron 87 $(74.3 \%)$ hombres y $68 \%$ de la población refirió antecedente de tabaquismo ( $p=0.87$ ). No se encontraron diferencias en las características basales entre ambos grupos de estudio según la edad, sexo, tabaquismo, sobrepeso-obesidad, diabetes tipo 2, hipertensión arterial sistémica, insuficiencia renal y hematuria macroscópica (Cuadro 1).

El diámetro del tumor primario fue mayor en el grupo de segunda revisión $(3 \pm 2$ vs $2.3 \pm 1.5$ $\mathrm{cm}, \mathrm{p}=0.03$ ), además de mayor prescripción de BCG intravesical coadyuvante (19 [45.2\%] vs 21 [28.8\%], p=0.07). De los pacientes a quienes se realizó segunda revisión, 23 tuvieron recurrencia (53.5\%) y 4 (9.3\%) evolución de la enfermedad, sin registrarse diferencias significativas en ambos grupos de estudio (Cuadro 2).

Se observó mayor porcentaje de tumor residual en pacientes con diagnóstico de cáncer de vejiga no invasor al músculo después de la resección transuretral inicial (39.5\%) e incremento en la estadificación $11.7 \%$ y grado tumoral (17.6\%) en la segunda revisión (Cuadro 3).

\section{DISCUSIÓN}

La resección transuretral de vejiga es un procedimiento diagnóstico decisivo en el tratamiento del cáncer de vejiga. No obstante, $45 \%$ de los pacientes con resección única tuvieron recurrencia en los primeros 12 meses de seguimiento. Este comportamiento puede deberse a factores como: tumores no detectados, resección incompleta, reimplantación de células tumorales después de
Cuadro 1. Características basales entre grupos de estudio

\begin{tabular}{|c|c|c|c|}
\hline & $\begin{array}{c}\text { Grupo } 1 \\
\text { (Con segunda } \\
\text { revisión) }\end{array}$ & $\begin{array}{l}\text { Grupo } 2 \\
\text { (Sin segunda } \\
\text { revisión) }\end{array}$ & $\mathbf{p}$ \\
\hline Edad & $\begin{array}{c}68.5 \pm 13.7 \\
(30-90)\end{array}$ & $\begin{array}{c}68.6 \pm 13.4 \\
(31-95)\end{array}$ & 0.97 \\
\hline \multicolumn{3}{|l|}{ Sexo } & \multirow[t]{3}{*}{0.65} \\
\hline Masculino & $33(76.7 \%)$ & $54(73 \%)$ & \\
\hline Femenino & $10(23.3 \%)$ & $20(27 \%)$ & \\
\hline \multicolumn{3}{|l|}{ Tabaquismo } & \multirow[t]{3}{*}{0.87} \\
\hline Sí & $29(67.4 \%)$ & $51(68.9 \%)$ & \\
\hline No & $14(32.6 \%)$ & $23(31.1 \%)$ & \\
\hline IMC* & $\begin{array}{c}24 \pm 4.66 \\
(19.6-38.2)\end{array}$ & $\begin{array}{c}25.9 \pm 3.8 \\
(17.8-35)\end{array}$ & 0.25 \\
\hline \multicolumn{3}{|l|}{ DM2** } & \multirow[t]{3}{*}{0.42} \\
\hline Sí & $10(23.3 \%)$ & $22(30.1 \%)$ & \\
\hline No & $33(76.7 \%)$ & $51(69.9 \%)$ & \\
\hline \multicolumn{3}{|l|}{ Dislipidemia } & \multirow[t]{3}{*}{0.03} \\
\hline Sí & $8(18.6 \%)$ & $28(38.4 \%)$ & \\
\hline No & 35 (81.4\%) & $45(61.4 \%)$ & \\
\hline \multicolumn{3}{|l|}{ Hipertensión } & \multirow[t]{3}{*}{0.60} \\
\hline Sí & $22(51.2 \%)$ & $41(56.2 \%)$ & \\
\hline No & $21(48.8 \%)$ & $32(43.8 \%)$ & \\
\hline \multicolumn{3}{|l|}{$\begin{array}{l}\text { Depuración de } \\
\text { creatinina }\end{array}$} & \multirow[t]{3}{*}{0.81} \\
\hline$<60$ & $12(33.3 \%)$ & $25(35.7 \%)$ & \\
\hline$\geq 60$ & $24(66.7 \%)$ & $45(64.3 \%)$ & \\
\hline \multicolumn{4}{|l|}{ Hematuria } \\
\hline $\begin{array}{l}\text { Microhema- } \\
\text { turia }\end{array}$ & $8(18.6 \%)$ & $11(14.9 \%)$ & 0.60 \\
\hline $\begin{array}{l}\text { Macrohema- } \\
\text { turia }\end{array}$ & 35 (81.4\%) & $63(85.1 \%)$ & \\
\hline
\end{tabular}

la intervención quirúrgica y aparición de novo en el urotelio de alto riesgo. ${ }^{10}$ Diferentes estudios y metanálisis demuestran que la aplicación de BCG y la resección transuretral representan un tratamiento conservador efectivo. ${ }^{11}$ Actualmente, el protocolo recomendado en estos pacientes es la estadificación adecuada, mediante la resección transuretral satisfactoria y seguimiento 
Cuadro 2. Características patológicas, coadyuvancia intravesical y desenlaces oncológicos

\begin{tabular}{|c|c|c|c|}
\hline & $\begin{array}{c}\text { Grupo } 1 \\
\text { (Con segunda } \\
\text { revisión) }\end{array}$ & $\begin{array}{c}\text { Grupo } 2 \\
\text { (Sin segunda } \\
\text { revisión) }\end{array}$ & $\mathbf{p}$ \\
\hline $\begin{array}{l}\text { Tamaño tumoral } \\
(\mathbf{c m})\end{array}$ & $3 \pm 2$ & $2.3 \pm 1.5$ & 0.03 \\
\hline \multicolumn{3}{|l|}{ Estadio T } & \multirow{4}{*}{0.15} \\
\hline $\mathrm{Ta}$ & $12(27.9 \%)$ & $34(45.9 \%)$ & \\
\hline $\mathrm{T} 1$ & $30(69.8 \%)$ & $39(52.7 \%)$ & \\
\hline Tis & $1(2.3 \%)$ & $1(1.4 \%)$ & \\
\hline \multicolumn{3}{|l|}{$\begin{array}{l}\text { Grado de } \\
\text { diferenciación }\end{array}$} & \multirow{4}{*}{0.55} \\
\hline G1 & $6(15.8)$ & $11(15.3 \%)$ & \\
\hline G2 & $13(34.2 \%)$ & $32(44.4 \%)$ & \\
\hline G3 & $19(50 \%)$ & $29(40.3 \%)$ & \\
\hline Mitomicina C & & & \multirow{3}{*}{0.60} \\
\hline Sí & $3(7.3 \%)$ & $5(6.8 \%)$ & \\
\hline No & 38 (92.7\%) & $68(93.2 \%)$ & \\
\hline \multicolumn{3}{|l|}{ BCG } & \multirow{3}{*}{0.07} \\
\hline Sí & $19(45.2 \%)$ & $21(28.8 \%)$ & \\
\hline No & $24(54.8 \%)$ & $52(71.2 \%)$ & \\
\hline $\begin{array}{l}\text { Seguimiento } \\
\text { (meses) }\end{array}$ & $23 \pm 32$ & $60 \pm 128$ & \\
\hline \multicolumn{3}{|l|}{ Recurrencia } & \multirow{3}{*}{0.95} \\
\hline Sí & $23(53.5 \%)$ & $40(54.1 \%)$ & \\
\hline No & $20(46.5 \%)$ & $34(45.9 \%)$ & \\
\hline \multicolumn{3}{|l|}{ Progresión } & \multirow{3}{*}{0.39} \\
\hline Sí & $4(9.3 \%)$ & $11(14.9 \%)$ & \\
\hline No & 39 (90.7\%) & $63(85.1 \%)$ & \\
\hline
\end{tabular}

* BCG: bacilo de Calmette-Guérin.

estrecho con cistoscopias trimestrales, en especial durante los primeros dos años, tomando en cuenta los pacientes de muy alto riesgo para considerar la cirugía radical.?

La resección inicial tiene función importante en la infraestadificación, incluso en la prevención de la recurrencia-persistencia. Herr y su grupo $^{12}$ analizaron 1,312 pacientes con tumores
Cuadro 3. Características histopatológicas de segunda revisión

\begin{tabular}{|c|c|}
\hline \multicolumn{2}{|c|}{ Tumor residual } \\
\hline Sí & 17 (39.5\%) \\
\hline No & $26(60.5 \%)$ \\
\hline \multicolumn{2}{|l|}{ Estadio $\mathrm{T}$} \\
\hline Ta & $6(1 Z 4 \%)$ \\
\hline $\mathrm{T} 1$ & $8(18.6 \%)$ \\
\hline $\mathrm{T} 2$ & $1(2.3 \%)$ \\
\hline $\mathrm{CIS}^{*}$ & $2(4.7 \%)$ \\
\hline Sin tumor & $26(60.5 \%)$ \\
\hline \multicolumn{2}{|l|}{ Grado } \\
\hline Bajo grado & $7(16.3 \%)$ \\
\hline Alto grado & $10(23.3 \%)$ \\
\hline Sin tumor & $26(60.5 \%)$ \\
\hline
\end{tabular}

papilares de vejiga, tratados en el Memorial Sloan-Kettering Cancer Center, y encontraron que $50 \%$ tuvo enfermedad residual, aunque sólo 5\% de alto grado, de los que 15\% tuvieron enfermedad invasora. En los pacientes con pT1, la re-estadificación a enfermedad con invasión al músculo fue diferente con base en la coexistencia de músculo en la primera pieza quirúrgica, y concluyeron que la resección inicial suele fallar al remover todo el tumor de la vejiga. En ese estudio, $18 \%$ de los pacientes tuvieron recurrencia después de un año y $81 \%$ de los tumores se encontraron en el sitio de la resección inicial. De acuerdo con estos resultados, las guías internacionales establecieron criterios y recomendaciones para el protocolo de segunda revisión. Aunque la resección transuretral de vejiga es un procedimiento de rutina y que no ha cambiado en las últimas décadas, nunca se han definido los criterios de calidad de la resección de forma clara y sistemática. Sin embargo, estas recomendaciones se basan, principalmente, en opiniones de expertos y limitados ensayos clínicos con asignación al azar, por lo que no se realizan de forma universal. 
Un estudio de cohorte internacional, en el que evaluaron 1,136 pacientes con carcinoma urotelial T1G3, tratados con cistectomía, reportó $51.4 \%$ de los casos con re-estadificación a T2 y $33.4 \%$ con enfermedad no confinada al órgano T3 o mayor con cualquier $\mathrm{N}$ (ganglios)..$^{13} \mathrm{En}$ nuestra serie se encontró tumor residual durante la segunda revisión en $39.5 \%$ de los casos, lo que coincide con lo reportado en la bibliografía (24-75.9\%); ${ }^{14,15}$ además, solo observamos un paciente con pT2 en la segunda revisión $(2.3 \%)$. La re-estadificación a enfermedad con invasión al músculo en las distintas series reportadas varía de 1.3 a $30 \% .^{16,17}$ Estos resultados pueden ser diferentes por la variabilidad interobservador entre el diagnóstico emitido por los patólogos (20-40\%), lo que tiene implicaciones pronósticas. ${ }^{18,19}$

En los pacientes con segunda revisión con tumor residual se observó aumento en la estadificación (pT) y el grado tumoral, en 2 (11.76\%) y $3(17.64 \%)$ pacientes, respectivamente. Entre los factores de riesgo para tumor residual en la segunda revisión se encontraron: tamaño del tumor inicial (más de $3 \mathrm{~cm}$ ), grado y coexistencia de tumores múltiples. ${ }^{20}$ Un análisis multivariado de 211 pacientes con cáncer de vejiga no invasor al músculo reportó que el subgrupo de alto riesgo (según EORTC) representó un factor independiente para la expresión del tumor en la segunda revisión (HR 12.65, IC95\%: 1.9382.75; $\mathrm{p}=0.008) .{ }^{21}$

En nuestro estudio, la mediana de seguimiento fue mayor a dos años y no se observaron diferencias en cuanto a la tasa de recurrencia y progresión de la enfermedad entre los grupos de estudio. Aunque parezca que el grupo de pacientes con segunda revisión tuvo enfermedad con comportamiento biológico más agresivo (pues el diámetro del tumor primario fue mayor [ $3 \pm 2$ vs $2.3 \pm 1.5 \mathrm{~cm} ; \mathrm{p}=0.03]$ ) y se encontró una tendencia hacia el uso de
BCG intravesical adyuvante (19 [45.2\%] vs 21 $(28.8 \%), p=0.07)$, no encontramos diferencias en los desenlaces oncológicos. Aunado a esto, consideramos que el diseño del estudio no fue el adecuado para calcular esta diferencia. Un ensayo clínico con asignación al azar, con 142 pacientes con diagnóstico reciente de carcinoma urotelial de vejiga no invasor al músculo y media de seguimiento de 32 meses, mostró que los pacientes sometidos a segunda revisión y que recibieron mitomicina $C$ coadyuvante, tuvieron mayor índice de supervivencia libre de recurrencia $(p=0.00)$, sin diferencia estadística en la tasa de progresión (4 vs $11.7 \%, p=0.09$ ), y supervivencia global (91.8 vs $89.7 \%, \mathrm{p}=0.73$ ). Los autores atribuyeron estos resultados a la alta tasa de tumor residual posterior a la primera resección transuretral de vejiga. ${ }^{22}$

Las principales limitantes de nuestro estudio fueron el tamaño de la muestra y su naturaleza retrospectiva. No obstante, la información acerca de la experiencia con este protocolo de tratamiento en México es escasa y no existen estudios publicados hasta la fecha. Se requieren estudios prospectivos adicionales para definir si esta intervención repercute en los desenlaces oncológicos y supervivencia global; sin embargo, por la alta tasa de tumor residual en nuestra serie y de acuerdo con lo publicado en todo el mundo, dejar de realizarla tendría implicaciones éticas importantes.

\section{CONCLUSIONES}

Existe un alto porcentaje de tumor residual en pacientes con diagnóstico de cáncer de vejiga no invasor al músculo con una resección transuretral de vejiga inicial, así como incremento en el estadio (pT) y grado tumoral en la segunda revisión. Con base en estos resultados, se justifica continuar con la práctica de segunda revisión en forma sistemática en México. 


\section{Financiamiento}

Los autores no recibieron ningún patrocinio para llevar acabo este estudio.

\section{Conflicto de intereses}

Jorge Mario Chamlati Cuello, Adrián Mauricio Garza Gangemi, Nezahualcóyotl Gonzaga Carlos y Emilio Iturriaga Goyon declaran no tener conflicto de interés. Ricardo Alonso Castillejos Molina: conferencista y colaborador de LillyIcos y Ferring.

\section{REFERENCIAS}

1. Ferlay J, Soerjomataram I, Ervik M, et al. Estimated cancer incidence, mortality and prevalence worldwide in 2012. GLOBOCAN 2012 v1.0: http://globocan.iarc.fr/Pages/ fact_sheets_population.aspx. Consultado el 23 de Abril de 2017.

2. Burger M, Catto JWF, Dalbagni G, et al. Epidemiology and Risk Factors of Urothelial Bladder Cancer. Eur Urol. 2013;63(2):234-241.

3. Gaya JM, Palou J, Cosentino $\mathrm{M}$, et al. A second transurethral resection could be not necessary in all high grade non-muscle-invasive bladder tumors. Actas Urol Esp. 2013;36(9): 539-44.

4. Jakse G, Algaba F, Malmström PU, et al. A Second-Look TUR in T1 Transitional Cell Carcinoma: Why? Eur Urol. 2004;45(5):539-546.

5. Turner W. T1G3 BladderTumours : The Case for Conservative Treatment. Eur Urol. 2004;45:401-405.

6. Baltaci S, Bozlu M, Yildirim A, et al. Significance of the interval between first and second transurethral resection on recurrence and progression rates in patients with high-risk non-muscle-invasive bladder cancer treated with maintenance intravesical Bacillus Calmette-Guerin. BJU Int. 2015;116(5):721-726.

7. Babjuk $M$, Böhle $A$, Burger $M$, et al. EAU Guidelines on Bladder. Cancer. 2016:1-45.

8. Divrik RT, Ahin AF, Yildirim Ü, et al. Impact of routine second transurethral resection on the long-term outcome of patients with newly diagnosed pT1 urothelial carcinoma with respect to recurrence, progression rate, and disease- specific survival: A prospective randomised clinical trial. Eur Urol. 2010;58(2):185-190.

9. Angulo JC, Palou J, García-tello A, et al. Second transurethral resection and prognosis of high-grade non-muscle invasive bladder cancer in patients not receiving Bacillus Calmette-Guérin . Actas Urol Esp. 2014;38(3):164-171.

10. Van der Heijden AG, Witjes JA. Recurrence, Progression, and Follow-Up in Non-Muscle-Invasive Bladder Cancer. Eur Urol Suppl. 2009;8(7):556-562.

11. Urdaneta G, Solsona E, Palou J. Intravesical Chemotherapy and BCG for the Treatment of Bladder Cancer : Evidence and Opinion. Eur Urol. 2008;7:542-547.

12. Herr HW, Donat SM. Quality control in transurethral resection of bladder tumours. BJUI. 2008:1242-1246.

13. Fritsche $H$, Burger $M$, Svatek RS, et al. Characteristics and outcomes of patients with clinical T1 grade 3 urothelial carcinoma treated with radical cystectomy: results from an international cohort. Eur Urol. 2010;57(2):300-309.

14. El-Barky E, Sebaey A, Eltabey M, et al. The importance of second-look transurethral resection for superficial bladder cancer. J Clin Urol. 2015;8(5).

15. Shim JS, Choi $\mathrm{H}$, Noh T II, et al. The clinical significance of a second transurethral resection for T1 high-grade bladder cancer : Results of a prospective study. Korean J Urol. 2015:429-434.

16. Herr HW. Role of re-resection in non-muscle-invasive bladder cancer. Sci World J. 2011:283-288.

17. Hashine $\mathrm{K}$, Ide T, Nakashima T, et al. Results of second transurethral resection for high-grade T1 bladder cancer. Urol Ann. 2016:10-15.

18. Giunchi F, Panzacchi R, Capizzi E, et al. Role of inter-observer variability and quantification of muscularis propria in the pathological staging of bladder cancer. Clin Genitourin Cancer. 2016;14(4):307-312.

19. Tosoni I, Wagner U, Sauter G, et al. Clinical significance of interobserver differences in the staging and grading of superficial bladder cancer. BJU Int. 2000;85(1):48-53.

20. Ali MH, Ismail IY, Eltobgy A, et al. Evaluation of secondlook transurethral resection in restaging of patients with nonmuscle-invasive bladder cancer. J Endourol. 2010;24(12):2047-2050.

21. Husillos-Alonso A, Rodríguez- Fernández E, Heranz Amo et al. MINERVA. Minerva Urol Nefrol. 2014;66(4):233-240.

22. Divrik RT, Yildirim Ü, Zorlu F, et al. The effect of repeat transurethral resection on recurrence and progression rates in patients with $\mathrm{t} 1$ tumors of the bladder who received intravesical mitomycin: a prospective, randomized clinical trial. J Urol. 2006;175(5):1641-1644. 